Article original

\title{
Hyperoxalurie primaire de type 1 : de l'enfance à l'âge adulte, comment gérer adéquatement l'adhésion au traitement médical ?
}

\section{Type 1 primary hyperoxaluria: From childhood to adult, how to manage adequately medical therapy compliance?}

\author{
Marie Leflot ${ }^{\mathrm{a}}$, Jean-Marie Krzesinski ${ }^{\mathrm{b}, *}$, Laure Collard $^{\mathrm{c}}$, Alexandre Thomas ${ }^{\mathrm{c}}$, \\ Marie-Sophie Ghuysen ${ }^{\mathrm{c}}$ \\ a Université de Liège, domaine universitaire B35, 4000, Liège, Belgique \\ bervice de néphrologie-dialyse-transplantation, CHU Sart-Tilman, domaine universitaire B35, 4000, Liège, Belgique \\ ${ }^{\mathrm{c}} \mathrm{CHU}$ de Liège, domaine universitaire $\mathrm{B35}, 4000$, Liège, Belgique
}

\section{N F O A R T I C L E}

\section{Historique de l'article:}

Reçu le 20 mars 2017

Accepté le 25 juin 2017

\section{Mots clés :}

Hyperoxalurie primaire de type 1

Lithiases rénales

Observance thérapeutique

Transplantation rénale et hépatique

\begin{abstract}
R É S U M É
Nous rapportons le cas de trois jeunes patients atteints d'hyperoxalurie primaire de type 1 , trouble métabolique d'origine génétique caractérisé par l'accumulation intracellulaire d'oxalate et pouvant résulter en une maladie rénale terminale avec atteinte systémique. Nous disposons actuellement de moyens thérapeutiques conservateurs efficaces si la prise en charge des enfants est précoce. Si l'efficacité de ce traitement rigoureux et exigeant a été démontrée, l'observance est un défi quotidien amplifié lorsque l'enfant avance en âge. La vérification de l'adhésion thérapeutique doit, par conséquent, rester une préoccupation majeure des professionnels de santé en charge de cette pathologie. Une meilleure compréhension des raisons de non-observance est nécessaire afin d'améliorer le suivi de ces patients chez qui l'éducation thérapeutique est une priorité.
\end{abstract}

(c) 2017 Société francophone de néphrologie, dialyse et transplantation. Publié par Elsevier Masson SAS. Tous droits réservés.

\section{A B S T R A C T}

We report the cases of three young patients suffering from type 1 primary hyperoxaluria, a metabolic genetic disorder characterized by intracellular accumulation of oxalate and which may result in endstage renal disease with systemic impairment. A number of effective conservative therapeutic means are available for early management of affected children particularly when he is growing older. Despite the demonstrated efficacy of conservative therapy, compliance represents a major and daily challenge. Monitoring therapeutic compliance is thus an important task for physicians in charge of this disease. A better understanding of non-compliance causes is required to improve the follow-up of patients for whom treatment education must be a priority.

(c) 2017 Société francophone de néphrologie, dialyse et transplantation. Published by Elsevier Masson SAS. All rights reserved.
Kidney and liver transplantation

Kidney stones

Type 1 primary hyperoxaluria

Therapeutic compliance

\section{Introduction}

L'hyperoxalurie primaire de type 1 (HP1) est une maladie rare héritée sur le mode autosomique récessif. Ce trouble inné du métabolisme du glyoxylate est lié au déficit d'une enzyme du

\footnotetext{
* Auteur correspondant.

Adresse e-mail : jm.krzesinski@chu.ulg.ac.be (J.-M. Krzesinski).
}

peroxisome hépatique, l'alanine glyoxylate aminotransférase (AGT). Le déficit de cette enzyme, dont le cofacteur est la pyridoxine, entraîne une surproduction hépatique d'acide oxalique, composé éliminé par le rein. Vu les concentrations élevées, l'oxalate urinaire se lie au calcium pour former des cristaux, lits des lithiases d'oxalate calcique [1].

Les manifestations cliniques sont hétérogènes et peuvent inclure une néphrocalcinose, des calculs urinaires récurrents avec 
les éventuelles complications obstructives et/ou infectieuses associées, une insuffisance rénale progressive avec dépôts systémiques d'oxalate dans les cas les plus sévères. L'oxalose systémique peut conduire à des anomalies diverses sévères (cardiaques, vasculaires, thyroïdiennes, visuelles, ostéo-articulaires) [2,3].

L'HP1 est plus fréquente dans les pays où les mariages consanguins sont tolérés. En Europe, sa prévalence est de 1/ 300000 à $1 / 10^{6}$. La rareté et la méconnaissance de cette pathologie rendent son diagnostic difficile. Les premiers symptômes sont rarement reconnus avant l'âge de 5 à 6 ans. L'insuffisance rénale chronique (IRC) survient entre 25 et 40 ans dans $50 \%$ des cas et représente moins de $2 \%$ des IRC terminales (IRCT) de l'enfant [4,5].

Seuls le dépistage et le traitement médical précoces permettent d'éviter ou de retarder l'insuffisance rénale et l'oxalose systémique.

Après de brefs rappels physiopathologiques et thérapeutiques, nous décrirons l'histoire clinique de trois patients et l'évolution actuelle du plus âgé d'entre eux. Ensuite, nous aborderons les difficultés importantes auxquelles les patients, leurs parents et les cliniciens se heurtent dès l'adolescence, et l'indispensable nécessité de remise en question des équipes médicales lorsque le patient ne veut plus ou ne peut plus adhérer au traitement.

\section{Physiopathologie}

L'oxalate provient principalement de deux sources : le métabolisme hépatique du glyoxylate endogène (75 \%) et l'alimentation (25\%). L'oxalate est éliminé uniquement par voie rénale. La production quotidienne d'oxalate est assez stable, de l'ordre de 4 à $8 \mathrm{mmol} / 1,73 \mathrm{~m}^{2}$ et, en présence de l'AGT, l'excrétion urinaire est faible ( $<0,55 \mathrm{mmol} / 1,73 \mathrm{~m}^{2}$ par jour) [6].

Le contrôle de l'absorption digestive dépend du contenu intestinal en calcium. En effet, seul l'ion oxalate libre est absorbé par l'intestin. S'il est complexé sous forme d'oxalate de calcium, il est peu résorbé et éliminé dans les selles. Il en résulte que toute diminution du calcium alimentaire dans la lumière intestinale augmente la quantité d'oxalate libre absorbé.

L'acide chlorhydrique, produit par les cellules gastriques, possède un pouvoir de dissolution des cristaux d'oxalate de calcium.

De plus, le microbiote intestinal est doté d'une bactérie capable de dégrader l'oxalate, l'Oxalobacter formigenes. L'abondance de cette bactérie au niveau du tractus digestif diminue l'excrétion urinaire d'oxalate [7].

En pratique, l'oxalate provient en majorité du métabolisme cellulaire endogène, raison pour laquelle les enfants souffrant de cette pathologie ne sont plus soumis à une diète restrictive en oxalate.

Dans l'HP1, le déficit en AGT hépatique (suite à la mutation du gène $A G X T$ ) entraîne une production massive d'oxalate par le foie, dépassant les capacités d'élimination rénale par défaut de transformation du glyoxylate en glycine et pyruvate, dérivés métaboliques solubles. L'oxalurie devient supérieure à $1 \mathrm{mmol} /$ $1,73 \mathrm{~m}^{2}$ par jour [8].

Plusieurs éléments sont responsables de la détérioration rénale progressive :

- la très faible solubilité de l'oxalate avec sursaturation des urines en cette substance très lithogène (lithiase formée de monohydrate d'oxalate de calcium) ;

- la précipitation puis les dépôts tubulaires et interstitiels d'oxalate calcique, avec inflammation et ensuite fibrose ;

- la formation de débris cellulaires favorisant la nucléation puis la fixation de nouvelles molécules d'oxalate et la cristallisation de novo, avec lésion rénale progressive et néphrocalcinose [9].

La toxicité rénale de l'oxalate sur les cellules tubulaires est actuellement mieux connue et est liée à l'activation de la phospholipase A2 et la production de lipides toxiques avec atteinte de la fonction mitochondriale, apoptose et nécrose cellulaire. Lorsque le débit de filtration glomérulaire (DFG) chute en dessous de $30 \mathrm{~mL} / \mathrm{min} / 1,73 \mathrm{~m}^{2}$, l'élimination urinaire de l'oxalate est proportionnellement réduite de sorte que l'oxalémie s'élève avec dépôts tissulaires et atteint une concentration supérieure à $50 \mu \mathrm{mol} / \mathrm{L}$ (pour une valeur normale $<10 \mu \mathrm{mol} / \mathrm{L}$ ).

Cependant, des facteurs environnementaux et génétiques créent une variabilité individuelle à cette dégradation rénale $[10,11]$.

\section{Traitement conservateur}

Le traitement actuel recommandé doit être " précoce et agressif ". Il a pour but d'éviter les épisodes de néphrolithiase et de retarder l'évolution vers l'insuffisance rénale avec oxalose systémique (Tableau 1). La pierre angulaire du traitement est l'hyperhydratation avec alcalinisation urinaire [12-14] :

- l'hyperhydratation orale inhibe la cristallisation. Elle est assurée par voie orale, avec parfois recours à un sondage nasogastrique ou pose de gastrostomie ;

- l'alcalinisation des urines, par des suppléments oraux de citrate de potassium, solubilise l'oxalate de calcium et inhibe la cristallisation ;

- la pyridoxine (vitamine B6), cofacteur de l'AGT, est bénéfique chez les patients atteints d'hyperoxalurie primaire de type 1 avec activité enzymatique résiduelle.

La corrélation génotype-phénotype n'étant pas encore connue pour les différentes mutations, la réponse thérapeutique est testée chez tous les patients sans attendre le résultat de l'analyse génétique. Une réduction du taux d'oxalate urinaire d'un facteur trois est une réponse positive à ce traitement $[15,16]$.

\section{Histoire clinique des patients}

Nous rapportons l'histoire de 3 enfants atteints d'HP1 ayant bénéficié d'un diagnostic et d'un traitement médical précoces, en association avec un investissement parental idéal. Les premières années, le traitement est optimal grâce à un entourage très attentif. En revanche, en grandissant, l'enfant acquiert de l'autonomie et peut s'opposer à ces mesures physiquement et psychologiquement très contraignantes.

\subsection{Première enfant}

La première enfant est âgée de 2 ans lorsqu'elle se présente pour des épisodes douloureux faussement attribués à des infections du tractus urinaire. La maman a constaté des dépôts de sables noirâtres dans les langes de l'enfant à plusieurs reprises, ce qui l'inquiète. Elle présente donc l'enfant à la consultation de néphrologie pédiatrique car elle doute du diagnostic et déplore que l'élément anamnestique de dépôts dans les couches n'ait pas été considéré. Les "infections urinaires "sont associées à des douleurs abdominales et une hématurie macroscopique. De nouvelles analyses urinaires sont donc réalisées, qui objectivent une hématurie avec leucocyturie, mais des cultures toujours stériles. Une mise au point complémentaire permet d'expliquer la raison des dépôts noirâtres dans les langes, à savoir des taux élevés d'oxalate urinaire, avec des ratios urinaires d'oxalate/créatinine et glycolate/créatinine respectivement de 5 et 6 fois la norme.

Des examens complémentaires sont réalisés, comprenant une échographie des voies urinaires, une scintigraphie rénale au DMSA 
Tableau 1

Stratégie du traitement conservateur.

\begin{tabular}{ll}
\hline Traitement conservateur & Dose recommandée \\
\hline Hyperhydratation orale & 3 à $5 \mathrm{~L} / \mathrm{m}^{2}$ de surface corporelle $24 \mathrm{~h} / 24$ \\
Citrate de potassium oral & 100 à $150 \mathrm{mg} / \mathrm{kg}$ de poids corporel \\
Pyridoxine & 5 à $10 \mathrm{mg} / \mathrm{kg} / \mathrm{j}$ \\
\hline
\end{tabular}

et une mesure isotopique du débit de filtration glomérulaire (DFG) par chrome 51 EDTA $\left({ }^{51} \mathrm{Cr}\right.$-EDTA).

L'échographie des reins montre une hyperéchogénicité diffuse, homogène et symétrique de la médullaire rénale, avec néphrocalcinose. La scintigraphie DMSA infirme le diagnostic de pyélonéphrite aiguë ou séquellaire. La scintigraphie fonctionnelle au ${ }^{51} \mathrm{CrEDTA}$ mesure un DFG à 78,6 mL/min/1,73 $\mathrm{m}^{2}$.

Un génotypage est réalisé, mettant en évidence une mutation homozygote c.716_718del pour le gène AGXT. Les parents, quant à eux, refusent d'être soumis à l'analyse moléculaire.

Le traitement instauré consiste en une hyperhydratation diurne et nocturne, l'administration de pyridoxine et l'alcalinisation des urines par du citrate de potassium que l'enfant accepte dans ses boissons sans sonde nasogastrique. Ce traitement est efficace puisque la petite fille, âgée maintenant de 4 ans, est devenue asymptomatique. Elle boit de l'eau citratée et est remarquablement observante. Les équipes paramédicales ont pu informer les enseignants de l'importance d'une hydratation continue. Néanmoins, la maman déplore un non-suivi et un déficit d'hydratation à l'école.

\subsection{Deuxième patient}

Le deuxième patient est actuellement âgé de 12 ans. Il a 3 ans au moment du diagnostic posé suite à l'émission de lithiases à répétition. Il est issu de parents non consanguins belge et italien. La symptomatologie est marquée par des troubles mictionnels : énurésie, incontinence et impériosité diurne conduisant à la réalisation d'examens complémentaires.

L'analyse urinaire montre un ratio urinaire oxalate/créatinine de 2 fois la norme. L'échographie de l'appareil urinaire objective des lithiases bilatérales et le génotypage réalisé montre que l'enfant est porteur de deux mutations du gène $A G X T$ : la mutation c.2_3delTGinsAT transmise par le père et la mutation c.508G $>$ A transmise par la mère.

Le traitement médical nécessite la mise en place d'une sonde nasogastrique puis d'une gastrostomie. Suite à une énurésie et des fuites urinaires diurnes, aggravées par l'hyperhydratation, le jeune adolescent présente une observance variable dans sa qualité et un mal-être compensé par une polyphagie avec obésité.

\subsection{Troisième enfant}

Le troisième patient, né de parents non consanguins, est âgé de 28 mois lorsqu'il est adressé à la consultation de néphrologie pédiatrique suite à l'émission douloureuse de 4 lithiases urinaires de taille variable, mais supérieure au centimètre. Il est à présent âgé de 20 ans.

L'analyse d'urines confirme la présence de cristaux d'oxalate de calcium, avec un ratio urinaire oxalate/créatinine de 3 fois la norme.

L'analyse morphologique des premières lithiases objective de l'oxalate de calcium. La clairance de la créatinine est estimée à $80 \mathrm{~mL} / \mathrm{min} / 1,73 \mathrm{~m}^{2}$ selon la formule de Schwartz. Le scanner abdominal montre la présence de lithiases rénales bilatérales, dans tous les groupes caliciels.

Sur base de ces données, un diagnostic d'hyperoxalurie primaire est évoqué.
L'enfant subit une biopsie hépatique avec confirmation diagnostique puisque l'activité de l'AGT résiduelle est mesurée à $3 \%$.

Les génomes du patient et de ses parents (tous deux asymptomatiques) sont également étudiés, avec recherche de mutation du gène AGTX. L'enfant est porteur des mutations c.9669$3 C>$ G héritée du père et $c .725 C>A$ (mutation non encore décrite dans la littérature) héritée de la mère.

Le traitement nécessite la mise en place d'une sonde de gastrostomie dès l'âge de 3 ans, permettant une hydratation et alcalinisation nocturne et diurne. L'évolution immédiate est optimale et ce, tant que la démarche thérapeutique est assurée par ses parents. La maman s'est totalement investie ayant compris l'évolutivité péjorative de la maladie rénale et la possibilité de retarder l'échéance d'une insuffisance rénale potentielle. Suite au départ à la retraite de la néphrologue pédiatrique du jeune patient, celui-ci n'est plus suivi par aucune équipe médicale entre l'âge de 9 et 14 ans, mais est pris en charge par sa maman de façon " exemplaire ", avec quelques épisodes de coliques néphrétiques annuels d'élimination spontanée non douloureuse, sans complication ni hospitalisation.

\section{5. Évolution}

Pour ces 3 observations, le diagnostic a été précoce et difficile, le traitement conservateur exigeant et ininterrompu, avec implication forte demandée aux parents.

La première patiente est actuellement âgée de 4 ans et présente une évolution tout à fait favorable en raison d'une adhésion complète au traitement.

Le deuxième patient a aujourd'hui 12 ans. Il présente depuis quelques mois une oxalurie en augmentation fluctuante en raison d'une diminution de la régularité face au traitement instauré. Il accepte de participer à différents " trainings " et études.

Le troisième patient est actuellement âgé de 20 ans et son histoire nous confronte au dilemme de la non-observance. Depuis quelques mois, il mobilise l'attention des équipes médicales. Enfant, il reste parfaitement équilibré, sans dégradation de fonction rénale car l'hyperhydratation et le traitement médical sont conduits par ses parents jour et nuit. Porteur d'une gastrostomie, il souffre de polyurie et énurésie, mais sa souffrance est également psychologique suite au regard moqueur des autres depuis l'humiliation par un professeur de gymnastique. L'entrée en adolescence est marquée par l'installation progressive d'un conflit familial autour de la maladie du jeune homme. Il exprime clairement ne plus pouvoir accepter le traitement proposé, puis imposé par ses parents jusqu'à ses 15 ans. Ce patient est révolté par sa maladie, d'autant qu'il compare sa vie à celle de son frère indemne, né après sélection embryonnaire.

L'évolution staturopondérale du jeune homme est excellente, avec une croissance staturale remarquable et une filtration glomérulaire préservée $\left(80 \mathrm{~mL} / \mathrm{min} / 1,73 \mathrm{~m}^{2}\right)$. Ceci est source de conflits supplémentaires vis-à-vis des parents qui ont remarquablement appliqué les consignes médicales malgré l'absence d'accompagnement médical et psychologique pendant plusieurs années.

Les actes manqués se répètent (prise excessive de médicaments et envies suicidaires). À 16 ans, la prise en charge en milieu intrahospitalier l'aide à exprimer ses attentes et son opposition au programme thérapeutique. Le jeune homme affirme son désir d'être guéri et donc transplanté, sa seule option actuelle de guérison. Il explique que l'arrêt de tout traitement pour " planter » ses reins lui permettra de convaincre ses parents et les équipes médicales d'accepter l'idée d'une transplantation hépatique.

Les bons résultats biologiques et urinaires ont un double impact contradictoire : s'ils rassurent l'équipe médicale et les parents dont 
l'option thérapeutique est le traitement conservateur optimal, ils sont interprétés par le patient comme un obstacle l'empêchant d'accéder au seul traitement qu'il souhaite, la transplantation hépatique.

Depuis 30 mois, il subit de nombreux épisodes de migrations lithiasiques extrêmement douloureux, des irradiations radiologiques répétées (43 radiographies de l'abdomen sans préparation et 19 tomodensitométries abdominales) et pas moins de 20 manœuvres urétérorénoscopiques sous anesthésie générale, de plus en plus difficiles, mettant sa vie en danger en raison des risques de complications par sepsis (Fig. 1).

Les traitements urologiques nécessitent des hospitalisations multiples (111 jours en 18 mois) avec déscolarisation. Malgré ce contexte médical défavorable pour lui, il persiste dans son idée de refus de se traiter correctement car, selon lui, " tout geste ne consistant pas en une transplantation ne le guérira pas et sera donc inutile ». Le jeune homme demande à l'équipe médicale et paramédicale de revoir ses propositions puisqu'il dit ne plus pouvoir continuer le traitement conservateur instauré.

Ce parcours de non-observance volontaire, les hospitalisations répétées pour prise en charge urologique, la douleur physique et psychique ont amené les équipes médicales à plusieurs confrontations multidisciplinaires. Le patient est depuis lors inscrit sur une liste de transplantation hépatique et/ou rénale combinée, mais la non-adhésion au traitement et l'évolution psychique vers un syndrome dépressif exigent une prise en charge rapprochée en psychiatrie.

\section{Alternative au traitement conservateur : la transplantation}

Trois possibilités de greffe sont discutées : la transplantation hépatique isolée, rénale isolée ou hépatorénale combinée. Cependant, la progression de la maladie rénale ne peut pas être anticipée car nous ne connaissons pas encore avec certitude toutes les corrélations génotype-phénotype [15].

La transplantation hépatique isolée permet de corriger le déficit enzymatique, et donc d'arrêter la production excessive d'oxalate. Elle implique une hépatectomie complète du foie natif. Dès lors, la transplantation hépatique isolée est rarement proposée, sauf pour des patients dont le DFG est supérieur à $60 \mathrm{~mL} / \mathrm{min} / 1,73 \mathrm{~m}^{2}$.

La transplantation rénale isolée a montré des résultats médiocres, avec une survie du greffon à 3 ans de 17 à $23 \%$ puisque le déficit enzymatique n'est pas corrigé. La perte du greffon est principalement liée aux dépôts d'oxalate dans le rein transplanté. Cette stratégie semble donc très discutable et envisageable uniquement pour les patients répondant à la pyridoxine et au traitement par hyperhydratation-alcalinisation $[17,18]$.

La transplantation rein et foie (combinée ou séquentielle) est proposée dès que le DFG est compris entre 40 et $60 \mathrm{~mL} / \mathrm{min} /$ $1,73 \mathrm{~m}^{2}$ et si le patient présente des urolithiases agressives récurrentes en dépit d'un traitement médical optimal [2].

La stratégie de transplantation est toujours discutée au cas par cas, mais aucun consensus ne se dégage actuellement. L'allocation de transplantation dont nous disposons en Belgique (Eurotransplant) ne requiert pas de limite de filtration glomérulaire pour qu'une greffe simultanée de foie et de rein soit envisageable. En revanche, un patient ayant bénéficié d'une transplantation hépatique ne peut être inscrit sur une liste d'attente pour une greffe rénale que si le DFG est inférieur à $15 \mathrm{~mL} / \mathrm{min} / 1,73 \mathrm{~m}^{2}$. Cette notion est importante dans l'HP1 dans la mesure où les dépôts d'oxalate systémiques se mobilisent après la transplantation hépatique isolée [18].

L'avantage d'une greffe combinée est d'obtenir des organes d'excellente qualité et de limiter le pool antigénique apporté par un seul donneur par rapport à une greffe séquentielle foie puis rein. Le but est de prévenir la récidive de la maladie au niveau de l'allogreffe rénale et de corriger le défaut métabolique par la transplantation hépatique [19-22].

\section{Autres perspectives}

Plusieurs thérapies expérimentales de l'HP1 sont en cours d'investigation : administration directe d'Oxalobacter formigenes sous forme capsulaire, transplantation néonatale d'hépatocytes, transfert du gène $A G X T$ utilisant un vecteur viral adéno-associé, stabilisateurs cinétiques de l'AGT, modulateurs des protéines de l'homéostasie, inhibition de la production du glyoxylate, précurseur de l'oxalate, blocage de l'oxydation du glycolate en glyoxylate. . . [2,16,17].

\section{Discussion à la lumière de ces trois observations}

Au début de la maladie, les mesures conservatrices sont essentiellement assurées par les parents après explications et formations thérapeutiques pratiques. Elles sont généralement
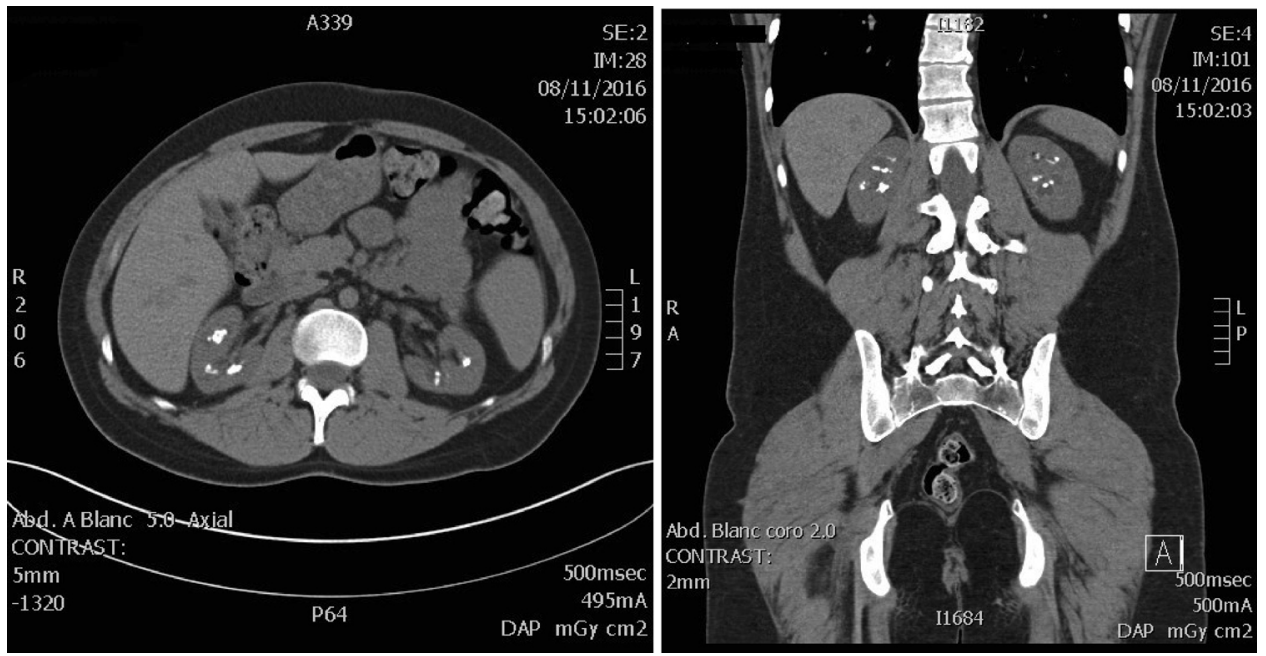

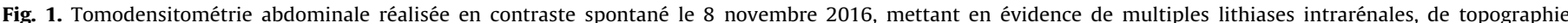

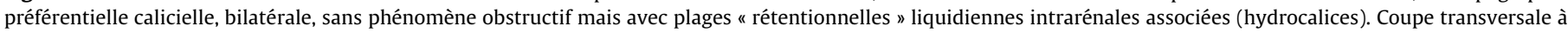
gauche et coupe frontale à droite. 
pénibles pour le jeune patient, car elles requièrent le plus souvent des gestes invasifs tels que la mise en place d'une sonde nasogastrique ou la pose d'une gastrostomie.

D'un point de vue psychologique, la pose d'une sonde ou d'un bouton de gastrostomie est un facteur de souffrance majoré lorsque l'enfant grandit : la scolarité, le regard des autres et les difficultés avec le corps enseignant aggravent ce malaise. À l'adolescence, le port de sondes nasogastrique ou de gastrostomie peut être source de moqueries et de limitations quotidiennes, par exemple dans les activités sportives ou sexuelles. Il en résulte des modifications de la perception de l'image corporelle, parfois à l'origine d'une perte de l'estime de soi, un sentiment de dévalorisation, voire de honte, une tendance au repli sur soimême et une révolte vis-à-vis des parents et du corps médical.

L'évolution du dernier patient, jeune homme sensé et obstiné, est particulièrement interpellante pour les équipes médicales, elles-mêmes divisées quant à la conduite à tenir. Après de multiples rencontres, au départ très tendues, avec et sans ses parents, le jeune adulte a pu exprimer plus calmement ce constat intolérable pour lui : être répondeur au traitement conservateur signifie qu'il ne peut être candidat à la transplantation, seule porte de sortie valable pour lui face à sa maladie. Dès lors, il refuse de se soigner pour pouvoir bénéficier d'une transplantation. Malgré une prise en charge par des urologues, néphrologues, chirurgiens de la transplantation et psychiatres, il décide de suivre la ligne de conduite de vie qu'il s'est tracée, après longue réflexion : le refus thérapeutique conservateur, de façon à faire admettre son idée de transplantation, même si cette voie lui impose des conflits (opposition avec ses parents et thérapeutes), des privations de liberté (hospitalisations) et des douleurs (coliques néphrétiques). Cette opposition farouche a finalement débouché sur un changement d'attitude des intervenants médicaux.

Le discours médical initial indiquait que la transplantation ne pouvait être proposée en raison d'une fonction rénale normale. Mais la demande insistante a permis d'envisager la proposition de transplantation, à condition de travailler l'adhésion au traitement médical et la gestion de l'anxiété. Les parents ont également modifié leur attitude et acceptent le choix thérapeutique de leur fils, différent de celui pour lequel ils ont déployé tant d'efforts. Le jeune homme a aussi mûri. Il dit avoir très bien compris les risques de morbi-mortalité inhérents à son choix. Il commence à comprendre les nécessités impérieuses de l'observance après transplantation, mais développe un syndrome dépressif majeur. La prise en charge psychiatrique depuis un an lui permet de revoir sa position.

L'histoire de ces trois patients d'âge croissant nous permet d'entrevoir les difficultés rencontrées :

- chez le petit enfant, la difficulté diagnostique et la culpabilité des parents sont suivies d'un investissement énorme avec traitement rigoureusement suivi ;

- ensuite, à l'adolescence, l'adhésion thérapeutique chute avec l'acquisition de l'autonomie ;

- et enfin, une alternative de proposition thérapeutique peut être finalement adoptée, comme pour le troisième patient.

\section{Conclusion}

L'observance thérapeutique est influencée au cours du temps par de nombreux paramètres, dont les aspirations du patient luimême, les caractéristiques de la maladie, les particularités de son traitement, les décisions et comportements du médecin, ainsi que la structure du système de soins du pays.

La non-observance thérapeutique peut avoir des répercussions graves sur le patient et l'évolution de sa maladie, avec un impact négatif sur les coûts des soins de santé.

Il est donc judicieux d'évaluer de manière rapprochée la concordance entre le souhait et le comportement du patient vis-àvis du traitement et les recommandations du médecin. Pour ce faire, il est préférable d'utiliser des méthodes impliquant les patients eux-mêmes dès le plus jeune âge, car l'observance apparaît comme le reflet du vécu d'une maladie chronique.

Cependant, face à un tel problème de non-adhésion, il n'existe pas de réponse ni de solution unique. L'aide du médecin traitant, de l'environnement familial et des autres partenaires de la santé est primordiale. Les réunions multidisciplinaires récurrentes représentent une pierre angulaire à la cohésion médicale, parfois mise en difficulté par un patient. Elles restent indispensables pour maintenir la confiance du patient, gage d'une acceptation optimale de sa prise en charge thérapeutique.

\section{Déclaration de liens d'intérêts}

Les auteurs déclarent ne pas avoir de liens d'intérêts.

\section{Références}

[1] Harambat J, Fargue S, Bacchetta J. Primary hyperoxaluria. Int J Nephrol 2011;2011:864580.

[2] Cochat P, Liutkus A, Fargue S. Primary hyperoxaluria type 1: still challenging! Pediatr Nephrol 2006;21:1075-81

[3] Gagnadoux M F.. Oxalose. EMC. Pédiatrie. Paris: Elsevier Masson SAS; 2004.

[4] Cochat P, Deloraine A, Rotily M. Epidemiology of primary hyperoxaluria type 1. Nephrol Dial Transplant 1995;10:3-7.

[5] Lieske JC, Monico CG, Holmes WS. International registry for primary hyperoxaluria. Am J Nephrol 2005;25:290-6.

[6] Cochat P. Primary hyperoxaluria type 1. Kidney Int 1999;55:2533-47.

[7] Abratt VR, Reid SJ. Oxalate-degrading bacteria of the human gut as probiotics in the management of kidney stone disease. Adv Appl Microbiol 2010;72:63-87.

[8] Cochat P, Hulton SA, Acquaviva C. Primary hyperoxaluria type 1: indications for screening and guidance for diagnosis and treatment. Nephrol Dial Transplant 2012;27:1729-36.

[9] Habbig S, Beck BB, Hoppe B. Nephrocalcinosis and urolithiasis in children. Kidney Int 2011;80:1278-91.

[10] Milosevic D, Rinat C, Batinic D. Genetic analysis-a diagnostic tool for primary hyperoxaluria type I. Pediatr Nephrol 2002;17:896-8.

[11] Leumann E, Hoppe B. Primary hyperoxaluria type 1: is genotyping clinically helpful? Pediatr Nephrol 2005:20:555-7.

[12] Cochat P, Hulton SA, Acquaviva C. Primary hyperoxaluria type 1: indications for screening and guidance for diagnosis and treatment. Nephrol Dial Transplant 2012;27:1729-36.

[13] Cochat P, Groothoff J. Primary hyperoxaluria type 1: practical and ethical issues. Pediatr Nephrol 2013;28:2273-81.

[14] Fargue S, Harambat J, Gagnadoux MF. Effect of conservative treatment on the renal outcome of children with primary hyperoxaluria type 1. Kidney Int 2009;76:767-73.

[15] Danpure CJ, Jennings PR, Fryer P. Primary hyperoxaluria type 1: genotypic and phenotypic heterogeneity. J Inherit Met Dis 1994;17:487-99.

[16] Ben-Shalom E, Frishberg Y. Primary hyperoxalurias: diagnosis and treatment. Pediatr Nephrol 2015;30:1781-91.

[17] Cochat P, Fargue S, Harambat J. Primary hyperoxaluria type 1: strategy for organ transplantation. Curr Opin Organ Transplant 2010;15:590-3.

[18] Kemper MJ. The role of preemptive liver transplantation in primary hyperoxaluria type 1 . Urol Res 2005;33:376-9.

[19] Ganschow R, Hoppe B. Review of combined liver and kidney transplantation in children. Pediatr Transplant 2015:19:820-6.

[20] Büscher R, Büscher AK, Cetiner M. Combined liver and kidney transplantation and kidney after liver transplantation in children: indication, postoperative outcome, and long-term results. Pediatr Transplant 2015;19:858-65.

[21] Grewal HP, Brady L, Cronin I. Combined liver and kidney transplantation in children. Transplantation 2000;70:100-5

[22] Scheinman JI. Liver transplantation in oxalosis prior to advanced chronic kidney disease. Pediatr Nephrol 2010;25:2217-22. 